\title{
Low Cost Spatially Resolved Diffuse Reflectance Spectrometry in the SWIR range
}

\author{
Ornella RIEHM ${ }^{\text {a }}$, Luc ANDRE a , Anabela DA SILVA ${ }^{\text {b }}$, Anne KOENIG ${ }^{\text {a }}$ \\ ${ }^{a}$ Univ. Grenoble Alpes, F-38000 Grenoble, France - CEA, LETI, MINATEC Campus, F-38054 Grenoble, France \\ ${ }^{b}$ Institut Fresnel, CNRS, Aix-Marseille Université, Ecole Centrale Marseille, Campus de Saint Jérôme, F13013 Marseille, France \\ riehmornella@gmail.com, luc.andre@,cea.fr
}

\begin{abstract}
The lasers used for the telecom industry associated with the focal plane embedded in LIDAR for automotive could pave the way to robust yet low cost Spatially Resolved Diffuse Reflectance Spectroscopy in the SWIR range.
\end{abstract}

(C) 2021 Ornella RIEHM, Luc ANDRE, Anabela DA SILVA, Anne KOENIG

\section{Introduction}

Over the two last decades, DRS in the SWIR range [900-2000 nm] showed great promises in the noninvasive measurement of sugar blended in a water based diffusing media. However, the robustness of the measurement deteriorates as one considers real biological tissue. In fact, biological tissues show strong heterogeneities in its multilayered composition, and multiple biological chromophores have signatures in the SWIR.

On the other hand, the telecom industry provided a large amount of coherent or incoherent infrared sources with associated optics at low cost. The development of autonomous automotive requiring eye safe infrared LIDARS accelerated the spread of low-cost SWIR sensitive focal plane. Associated with srDRS postprocessing, multiple SWIR lasers with sensitive focal plane arrays could enhance the monitoring of the chromophore of interest [1].

We divided our work into 3 phases. At first, we made a comprehensive review of the work published on DRS in the SWIR range, and on the optical properties of biological tissues. Then, we decided on a two-pronged approach including the development of a high-end setup and a wearable. Finally, we summarized our spectral characterization, starting with our transmission results and pursuing with characterization in a diffusing media.

\section{Review of DRS in the SWIR range}

\section{$2.1 \quad$ Published and commercial setups}

For the beginning of our project, we investigated the different set-ups and devices found in the literature, and that used DRS for detecting glucose non-invasively. The setup used by the team of Pr. Katsuhiko Maruo [2] for example, is one that works in reflection: a halogen lamp emits a near infrared light that is incident on the sample, via an optical fiber bundle. On the same side of the sample (in fact, in the center of the bundle) is a detection fiber that collects the light reflected from the sample, which is then subjected to spectroscopy, and detected by an array photosensor.

Several portable devices have also been prototyped based on NIR spectroscopy. For example, several countries approved the use of the non-invasive TensorTip Combo Glucometer designed by Cnoga Medical Ltd, Israel [3]. It is composed of 4 LEDs $(600 \mathrm{~nm}-1000 \mathrm{~nm})$ and a color camera sensor that registers the light that goes through the fingertip. Glucose quantitative data is derived from the analysis of the transmitted light, and is reported to be efficiently measured for concentrations ranging from 70 to $440 \mathrm{mg} / \mathrm{dL}$. World Global Network, with Hello Extense [4], or RSP Systems, with the Wizmi prototype [5], also worked on the non-invasive monitoring of sugar levels.

Although none of these devices can be used to accurately dose insulin, and although they all require to be calibrated with an invasive measure prior to the non-invasive measure, they still represent a great progress on the road of a non-invasive glucose monitoring portable device.

\section{$2.1 \quad$ Optical properties of biological tissues}

The Beer-Lambert law of absorption states that there is a linear relationship between the concentration of the absorbing species contained in a solution, and the absorbance of the solution. By measuring the absorbance of a solution at an appropriate wavelength, we are thus able to deduct the concentration of the species of interest.

However, there are important challenges to the non-invasive measure of glucose in the derma or in the blood. Indeed, several species existing in these biological tissues absorb light in the NIR region. Particularly, water is responsible for an important part of light absorption over these wavelengths. As the human body is highly concentrated in water and biological tissues are very poorly concentrated in glucose, minimizing effects of light 
absorption by water is of the utmost importance. Although light absorption by glucose is higher is the range 2000-2400 nm, light absorption by water is lower at smaller wavelengths (i.e. $800 \mathrm{~nm}-1650 \mathrm{~nm}$ ). Working in the SWIR would minimize the light absorption by water, and thus enhance the selectivity to glucose molecules [6].

To separate the absorption due to water from which due to glucose, it could be interesting to select a wavelength at which light is only absorbed by water, and a wavelength at which light is absorbed by both water and glucose molecules [6]. This method could be expended to more than two light sources, to account for the overlapping peaks of other biological molecules (fats, proteins, hemoglobin). Glucose quantitative data can then be retrieved by algorithmic methods, such as the partial least square method [7] or principal component regression.

Finally, the presence of scattering molecules in the biological medium of interest results in a non-linear relationship between absorption spectra and concentration of absorbing components [8], a difficulty which must be taken into consideration.

\section{3. srDRS in the SWIR range development approach}

\subsection{Setup description}

Our setup is composed of a halogen lamp emitting a near-infrared light which is selected wavelength by wavelength by a monochromator. At the output of the monochromator, the light is guided via an optical fiber which is collimated to a second optical fiber. Between the two fibers is a cuvette made of Optical Glass, containing the sample to be studied. The transmitted light is then detected by an InGaAs detector.

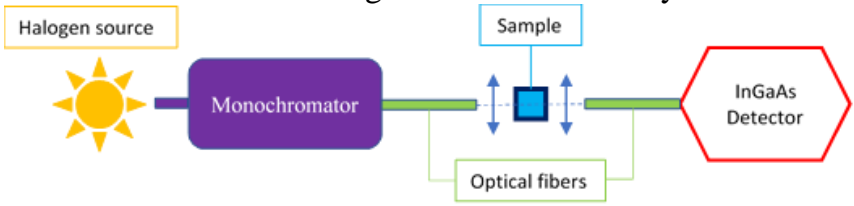

Fig. 1. Diagram of the experimental setup

\subsection{Methodology}

Before considering both the complexitiy of biological tissue and the integration of a "proof of concept", we must characterize the spectral signatures of the most important chromophores: water and glucose. Moreover, acquiring the absorbance of purified water has the double advantage of validating the setup described in 3.1, as the evolution of the absorption coefficient of water between $800 \mathrm{~nm}$ and $1650 \mathrm{~nm}$ is well documented in the literature. We also compared the experimental setup measurements with those obtained from our own FTIR.

The reference spectrum for this measurement being that of the cuvette filled only with air, we had to account for the Fresnel reflections at the interfaces between the different media. Furthermore, as Fresnel coefficients depend on the refraction indices of the different materials found on the light path, consequently, the knowledge of the refraction indices of the purified water and glucose solutions is fundamental for our future experiments. The indices of the solutions were measured experimentally with an Abbe refractometer. We traced the evolution of the refraction index of glucose solutions at room temperature, according to the glucose concentration in solutions.

To get a better understanding of the evolution of the absorption coefficient of glucose over the wavelengths of interest, we first studied it for concentrations much higher than the physiological values of healthy subjects (i.e. 70-110 mg/dL). We prepared three solutions at concentrations 1-2-3 g/dL.

Water displacement is the phenomenon by which the molecules of a solute take the place of water molecules, resulting in a decrease of the absorption due to water molecules only [9]. At concentrations such as $1 \mathrm{~g} / \mathrm{dL}$ and more, this phenomenon is no longer negligible and must be accounted for. As a consequence, we corrected this phenomenon via two methods found in the literature, that we adapted to our own results.

\section{First spectral resultsTransmission measurement}

We experimentally obtained the absorption coefficient of purified water, with the monochromator and the FTIR setup, and compared our data with that found in the literature [10-11]. Although the absorption peaks found experimentally with the monochromator are not as high as those found with the FTIR and in other scientific reports, this discrepancy is repeatable and can be accounted for. Moreover, the wavelengths at which the peaks occur are the same for all four setups: $1460 \mathrm{~nm}$.

Once we validated our results, we could measure the absorption coefficient of glucose. Before processing the data, the glucose absorption coefficient for concentrations $1 \mathrm{~g} / \mathrm{dL}, 2 \mathrm{~g} / \mathrm{dL}$ and $3 \mathrm{~g} / \mathrm{dL}$, presented negative values over the wavelength range of interest. These negative values translate as the direct influence of water displacement. Figure 2 shows the glucose absorption coefficient obtained for three different concentrations, after accounting for water displacement. This graph highlighted several bands of interest where the absorption 
coefficients of glucose are linear in function of the concentration. These bands are notably centered on $920 \mathrm{~nm}$, $1400 \mathrm{~nm}$ and $1550 \mathrm{~nm}$, which correspond to the glucose absorption bands reported in the literature [12].

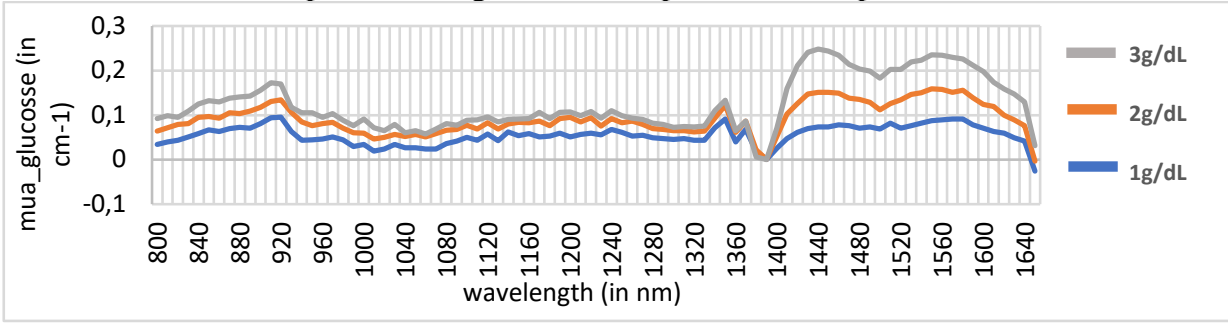

Fig. 2. Glucose absorption coefficients after post-processing

\subsection{Measurements in diffusing media}

Contrary to glucose aqueous solutions, biological tissues such as the derma or the capillaries are highly diffusing media in the SWIR. In order to get an idea of the evolution of the reflected light intensity in the derma vs the distance to the source of illumination, we performed Monte Carlo simulations, at the wavelengths that had been identified from the experiments. To perform these simulations, the absorption coefficients corresponding to hyper and hypo glycaemia (i.e. $110 \mathrm{mg} / \mathrm{dL}$ and $70 \mathrm{mg} / \mathrm{dL}$ ) were experimentally measured as presented above, and the anisotropic coefficient of human skin and the skin scattering properties was found in the literature.

This first approach showed a difference between the reflected light intensity in the case of hyper and hypo glycaemia: the non-invasive optical distinction between hyper and hypo-glycaemia levels, appears to be possible in the SWIR.

\section{Conclusion}

The srDRS technique is amongst the most promising methods for the non-invasive detection of glucose. The finality of this project is to obtain a portable sensor that could be used for daily non-invasive glucose monitoring. This miniaturization will be guided by the results of Monte Carlo simulations that will help us determine the optimum illumination and detection geometry, as well as the most appropriate wavelengths to be used for glucose detection. Focusing on the SWIR region could also lead to the development of a low-cost array of Germanium photodiodes to replace the InGaAs sensor we currently use in our bulky experimental setup.

\section{References}

[1] Anne Koenig, Nils Petitdidier, et al, "Contact, high-resolution spatial diffuse reflectance imaging system for skin condition diagnosis: a first-in-human clinical trial”; J. Biomed. Opt. 26(1) 012706 (29 January 2021) https://doi.org/10.1117/1.JBO.26.1.012706

[2] Maruo, Katsuhiko \& Tsurugi, Mitsuhiro \& Tamura, Mamoru \& Ozaki, Yukihiro. (2003). In Vivo Noninvasive Measurement of Blood Glucose by Near-Infrared Diffuse-Reflectance Spectroscopy. Applied spectroscopy. 57. 1236-44. 10.1366/000370203769699090.

[3] Segman, Y. (Joseph). (2018). Device and Method for Noninvasive Glucose Assessment. Journal of Diabetes Science and Technology, 12(6), 1159-1168. https://doi.org/10.1177/1932296818763457

[4] https://website.worldgn.com/heloextense/. Site accessed 16/02/2021.

[5] Hadar E., Chen R., Toledano Y., Tenenbaum-Gavish K., Atzmon Y., Hod M. (2019). Noninvasive, continuous, real-time glucose measurements compared to reference laboratory venous plasma glucose values. J. Matern. Fetal Neonatal Med. 32(20):3393-3400.

[6] N. Mert Vural, Yigit Yoleri, Hamdi Torun, "On feasibility of near-infrared spectroscopy for noninvasive blood glucose measurements," Proc. SPIE 10885, Opt. Diag. and Sensing XIX: Toward Point-of-Care Diagnostics, 108850R (2019) https://doi.org/10.1117/12.2503852

[7] Goodarzi, M.; Sharma, S.; Ramon, H.; Saeys, W. (2015) Multivariate calibration of NIR spectroscopic sensors for continuous glucose monitoring. TrAC Trends Anal. Chem., 67, 147-158.

[8] Alexeeva, N. V., \& Arnold, M. A. (2010). Impact of Tissue Heterogeneity on Noninvasive Near-Infrared Glucose Measurements in Interstitial Fluid of Rat Skin. Journal of Diabetes Science and Technology, 4(5), 1041-1054. https://doi.org/10.1177/193229681000400503

[9] Jung Y, Hwang J. "Near-Infrared Studies of Glucose and Sucrose in Aqueous Solutions: Water Displacement Effect and Red Shift in Water Absorption from Water-Solute Interaction”, Applied Spectroscopy. 67(2):171-180. 2013. doi:10.1366/12-06635

[10] K.F. Palmer and D. Williams, "Optical Properties of water in the near infrared”, Journal of the Optical Society of America, V.64, pp. 1107-1110, August, 1974

[11] G.M. Hale and M.R. Querry, "Optical Constants of Water in the 200-nm to 200- $\mu$ m Wavelength Region,"Appl. Opt. 12, 555-563, 1973

[12] Omar S Khalil, "Spectroscopic and Clinical Aspects of Noninvasive Glucose Measurements", Clinical Chemistry, Volume 45, Issue 2, Pages 165-177, 1 February 1999, https://doi.org/10.1093/clinchem/45.2.165 